With compliments of the Author 


\title{
A Convenient Synthesis of Tri- and Tetramethylbenzaldehydes from Readily Available Phenols
}

\author{
Persis Dhankher, Tom D. Sheppard* \\ Department of Chemistry, University College London, 20 Gordon St, London, WC1H 0AJ, UK \\ E-mail: tom.sheppard@ucl.ac.uk \\ Received: 08.10.2013; Accepted after revision: 28.10.2013
}

\begin{abstract}
This letter describes a convenient synthesis of the six isomeric tri- and tetramethylbenzaldehydes, which are not readily available from major chemical suppliers. Formylation of readily available phenols via electrophilic aromatic substitution provides compounds containing the correct aromatic substitution pattern. Suzuki cross-coupling of the corresponding trifluoromethanesulfonates with methylboronic acid then provides the benzaldehydes as single isomers.
\end{abstract}

Key words: aldehydes, palladium, Suzuki, aromatic compounds, formylation

Substituted benzaldehydes are important starting materials for use in a variety of chemical processes and they are frequently employed in medicinal chemistry to introduce substituted aryl groups into potential drug molecules. The introduction of multiple methyl groups onto the benzene ring can serve to alter the preferred three-dimensional conformation of the aryl unit in the molecule, leading to subtle changes in chemical and biological properties. ${ }^{1}$ There are 19 different benzaldehydes incorporating one or more methyl substituents on the aromatic ring and 13 of these compounds are available commercially in gram quantities from major suppliers. ${ }^{2}$ The remaining compounds 1a-f (Figure 1) are not available from major commercial suppliers, so typically they must be synthesized from more readily available aryl precursors. Aldehydes 1a-f have previously been synthesized by a variety of different routes including direct formylation of polymethylated benzenes, ${ }^{3}$ side-chain oxidation of polylmethylated benzenes, ${ }^{4}$ Grignard reaction of an aryl bromide with a suitable electrophile, ${ }^{5}$ partial reduction of carboxylic acid derivatives, ${ }^{6}$ or degradation of bicyclic systems. ${ }^{7}$ In the latter three cases the starting materials used are often as complex to prepare as the target aldehyde itself. Whilst direct formylation of a polymethylated benzene potentially offers a very direct route, polymethyl benzenes are prone to acid-catalyzed isomerization processes during the harsh conditions often needed for electrophilic aromatic substitution reactions. ${ }^{8}$ This can lead to complex mixtures of regioisomeric products which can be extremely difficult to separate.

SYNLETT 2014, 25, 0381-0384

Advanced online publication: 04.12 .2013

DOI: 10.1055/s-0033-1340302; Art ID: ST-2013-D0950-L

C Georg Thieme Verlag Stuttgart · New York<smiles>Cc1cc(Br)cc(C=O)c1C</smiles><smiles>Cc1ccc(C)c(C=O)c1C</smiles><smiles>Cc1cc(C=O)cc(I)c1C</smiles><smiles>Cc1ccc(C=O)c(C)c1C</smiles><smiles>Cc1cc(C=O)c(C)c(C)c1C</smiles><smiles>Cc1cc(C)c(C=O)c(C)c1C</smiles>

Figure 1 Tri- and tetramethylbenzaldehydes that are not readily available commercially

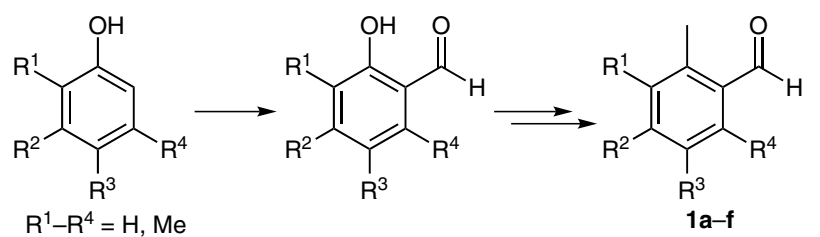

Scheme 1 Proposed synthetic route to tri- and tetramethylbenzaldehydes

As part of an ongoing medicinal chemistry project, we required access to all of the 19 methylated benzaldehyde isomers to explore a complex structure-activity relationship. In order to prepare all of the six isomers shown in Figure 1, we have developed a new synthetic route to enable all of these compounds to be prepared from cheap, commercially available phenols.

We envisaged that the desired aldehydes could potentially be synthesized by formylation of a phenol in an electrophilic aromatic substitution reaction, followed by crosscoupling of the phenol-derived sulfonate ester with methylboronic acid (Scheme 1). The phenol group would serve to activate the aromatic ring towards electrophilic aromatic substitution, preventing competing isomerization processes, and direct the substitution reaction to provide the desired product isomer. After isolation of the desired regioisomer, the phenol can then be converted into a methyl group via the corresponding trifluormethanesulfonate ester. The phenols $\mathbf{2 a}-\mathbf{e}$ required for the synthesis of aldehydes $1 \mathbf{a}-\mathbf{e}$ are all commercially available. In the case of aldehyde 1f, a suitable dihydroquinone precursor $\mathbf{2 f}$ containing the required substitution pattern is commercially available (vide infra). 
The formylation of phenols $\mathbf{2} \mathbf{a}-\mathbf{e}$ was carried out using dichloromethyl methyl ether and aluminium trichloride at room temperature (Scheme 2). ${ }^{9}$ Phenol 2a underwent formylation in good yield to give aldehyde $\mathbf{3}$, which was converted into triflate $\mathbf{4}$ under standard conditions. The formylation of phenol $\mathbf{2 b}$ under similar conditions gave a mixture of isomeric aldehydes where the major product was the undesired para-substitution product. Nevertheless, the isomers could readily be separated by chromatography, and aldehyde $\mathbf{5}^{9}$ was subsequently converted into aryl trifluoromethanesulfonate $\mathbf{6} .{ }^{10}$ Phenol $\mathbf{2 c}$ underwent formylation in good yield to give aldehyde $7^{11}$ which was in turn converted into triflate $\mathbf{8}^{12}$ The formylation of phenol 2d gave a mixture of isomeric aldehydes $9^{13}$ and $10^{14}$ which was converted into a mixture of the two corresponding triflates $\mathbf{1 1}{ }^{13}$ and $\mathbf{1 2} .^{14}$ This mixture of isomers is inconsequential as both triflates $\mathbf{1 1}$ and $\mathbf{1 2}$ will ultimately be converted into the same aldehyde 1d upon Suzuki coupling with methylboronic acid. Phenol 2 e provides no regioselectivity issues in the aromatic substitution reaction, and aldehyde $13^{15}$ and triflate 14 were synthesized without difficulty.

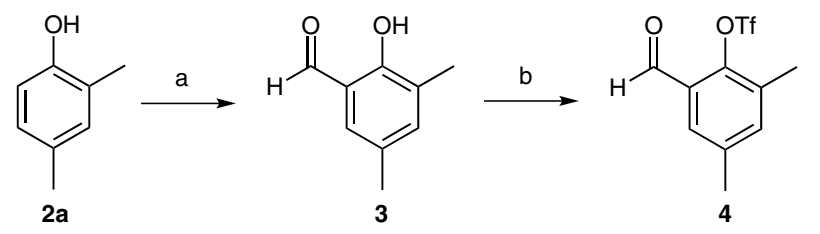<smiles>Cc1ccc(C)c(O)c1</smiles><smiles>[2H]c1cc(C=O)cc(C)c1O</smiles>

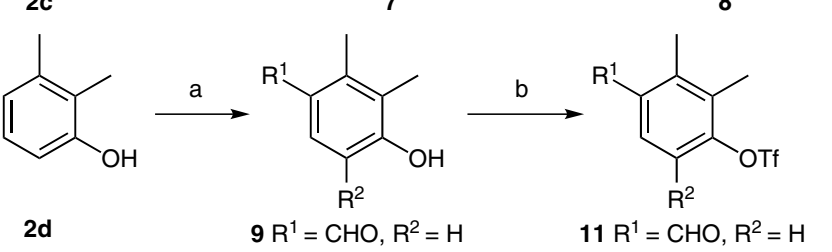

2d

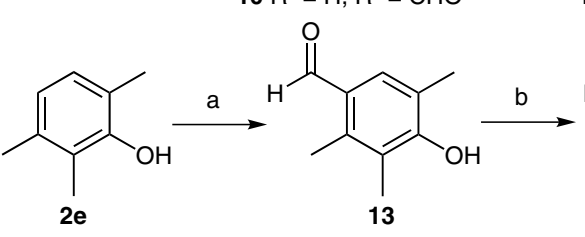

Scheme 2 Forymlation of starting phenols and subsequent conversion into the corresponding aryl trifluoromethanesulfonates. Reagents and conditions: a) $\mathrm{Cl}_{2} \mathrm{CHOMe}, \mathrm{AlCl}_{3}, \mathrm{CH}_{2} \mathrm{Cl}_{2}$, r.t., $65 \%$ (3), 15\% (5), $71 \%$ (7), 44\% (9 and 10), 60\% (13); b) $\mathrm{Tf}_{2} \mathrm{O}, \mathrm{Et}_{3} \mathrm{~N}, \mathrm{CH}_{2} \mathrm{Cl}_{2}, 64 \%$ (4), 34\% (6), 53\% (8), 95\% (11 and 12), 46\% (14).

With the desired triflates in hand, we then turned our attention to the proposed Suzuki cross-coupling with methylboronic acid (Scheme 3 and Table 1). The cross-coupling of methylboronic acid with aryl triflates has previously been reported under a variety of conditions. We evaluated several protocols for the conversion of triflate 4 into aldehyde 1a. ${ }^{16}$ With $\mathrm{Pd}\left(\mathrm{PPh}_{3}\right)_{4}$ as the catalyst, negligible conversion into the desired product was observed using either $\mathrm{K}_{2} \mathrm{CO}_{3}$ or $\mathrm{K}_{3} \mathrm{PO}_{4}$ as the base. Upon switching to $\mathrm{PdCl}_{2}(\mathrm{dp}-$ pf), however, excellent conversion into the desired product 1a was observed. ${ }^{16 \mathrm{~d}}$ With optimized conditions for the Suzuki cross-coupling in hand we went on to complete the synthesis of the aldehydes 1a-e (Table 1).

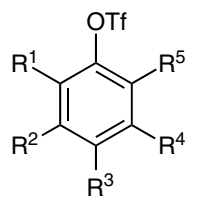

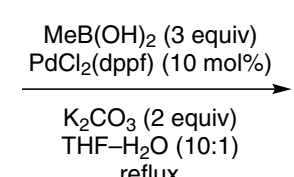

$\mathrm{R}^{1}, \mathrm{R}^{2}, \mathrm{R}^{4}=\mathrm{H}, \mathrm{Me}$

$\mathrm{R}^{3}=\mathrm{H}, \mathrm{Me}, \mathrm{CHO}$

$\mathrm{R}^{5}=\mathrm{CHO}, \mathrm{Me}$

Scheme 3 Suzuki cross-coupling of trifluoromethanesulfonates with methylboronic acid

Table 1 Suzuki Cross-Coupling Reactions

\begin{tabular}{llll}
\hline Entry & Trifluoromethanesulfonate & Product & Yield (\%) \\
\hline 1 & $\mathbf{4}$ & $\mathbf{1 a}^{17}$ & 99 \\
2 & $\mathbf{6}$ & $\mathbf{1 b}^{18}$ & 99 \\
3 & $\mathbf{8}$ & $\mathbf{1 c}^{6 \mathrm{a}}$ & 61 \\
4 & $\mathbf{1 1}$ and $\mathbf{1 2}$ & $\mathbf{1 d}^{19}$ & 97 \\
5 & $\mathbf{1 4}$ & $\mathbf{1 e}^{3 \mathrm{c}}$ & 80 \\
\hline
\end{tabular}

The remaining aldehyde 1f was synthesized from commercially available dihydroquinone $\mathbf{2 f}$ via trifluoromethanesulfonate formation and cross-coupling to give ester 15 (Scheme 4). ${ }^{20}$ An unoptimized reduction-oxidation sequence then provided aldehyde $\mathbf{1 f}^{3 \mathrm{a}}$ in moderate yield.<smiles>COC(=O)c1c(C)cc(O)c(C(=O)OC)c1C</smiles>

Scheme 4 Reagents and conditions: a) $\mathrm{Tf}_{2} \mathrm{O}, \mathrm{Et}_{3} \mathrm{~N}, \mathrm{CH}_{2} \mathrm{Cl}_{2}, 98 \%$; b) $\mathrm{MeB}(\mathrm{OH})_{2}, \mathrm{~K}_{2} \mathrm{CO}_{3}, \mathrm{PdCl}_{2}$ (dppf), THF- $\mathrm{H}_{2} \mathrm{O}$, reflux, $87 \%$; c) DIBAL-H, PhMe-Et $2 \mathrm{O}, 63 \%$; d) DMSO, $\mathrm{COCl}_{2}$, then $\mathrm{Et}_{3} \mathrm{~N}, \mathrm{CH}_{2} \mathrm{Cl}_{2}$, $18 \%$.

In conclusion, we have developed a general synthetic route to tri- or tetramethylbenzaldehydes from readily available phenols. Electrophilic aromatic formylation ${ }^{21}$ of a phenol provides access to compounds with the required substitution pattern as single isomers. Conversion into the 
corresponding triflate 22 and Suzuki cross-coupling with methylboronic acid $^{23}$ furnishes the target tri- and tetramethylbenzaldehydes as single isomers.

\section{Acknowledgment}

We would like to thank the University College London Drug Discovery $\mathrm{PhD}$ Program for supporting this work through the award of a studentship (to P.D.) and Professor Neil Millar (UCL Pharmacology) for helpful discussions.

Supporting Information for this article is available online at http://www.thieme-connect.com/ejournals/toc/synlett. Included are experimental procedures and ${ }^{1} \mathrm{H}$ and ${ }^{13} \mathrm{C}$ NMR spectra for all compounds.

\section{References and Notes}

(1) Brameld, K. A.; Kuhn, B.; Reuter, D. C.; Stahl, M. J. Chem. Inf. Model. 2008, 48,1 .

(2) All three methlybenzaldehydes and all six dimethylbenzaldehydes can be purchased from major commercial suppliers; 2,4,5-trimethylbenzaldehyde, 2,3,6-trimethylbenzaldehyde, 2,3,5,6-tetramethylbenzaldehyde, and pentamethylbenzaldehyde are also readily available.

(3) (a) Olah, G. A.; Kuhn, S. J. J. Am. Chem. Soc. 1960, 82, 2380. (b) Niedzielski, E. L.; Nord, F. F. J. Org. Chem. 1943, 8, 147. (c) Tanaka, M.; Fujiwara, M.; Ando, H. J. Org. Chem. 1995, 60, 3846. (d) Smith, L. I.; Agre, C. L. J. Am. Chem. Soc. 1938, 60, 648. (e) Fuson, R. C.; Southwick, P. L.; Rowland, S. P. J. Am. Chem. Soc. 1944, 66, 1109.

(4) (a) Blackstock, D. J.; Fischer, A.; Richards, K. E.; Wright, G. J. Aust. J. Chem. 1973, 26, 775. (b) Hunziker, E.; Myhre, P. C.; Penton, J. R.; Zollinger, H. Helv. Chim. Acta 1975, 58, 230.

(5) (a) Smith, L. I.; Nichols, J. J. Org. Chem. 1941, 6, 489. (b) Mannschreck, A.; Hartmann, E.; Buchner, H.; Andert, D. Tetrahedron Lett. 1987, 28, 3479.

(6) (a) Gaspar, P. P.; Hsu, J.-P.; Chari, S.; Jones, M. Jr. Tetrahedron 1985, 41, 1479. (b) Benington, F.; Morin, R. D.; Clark, L. C. Jr. J. Org. Chem. 1960, 25, 1542. (c) Smith, D. G.; Smith, D. J. H. J. Chem. Soc., Chem. Commun. 1975, 459.

(7) Matsubara, Y.; Takekuma, S.-I.; Yokoi, K.; Yamamoto, H.; Nozoe, T. Bull. Chem. Soc. Jpn. 1987, 60, 1415.

(8) (a) McCaulay, D. A.; Lien, A. P. J. Am. Chem. Soc. 1952, 74, 6246. (b) Allen, R. H. J. Am. Chem. Soc. 1960, 82, 4856. (c) Olah, G. A.; Meyer, M. W.; Overchuk, N. A. J. Org. Chem. 1964, 29, 2313. (d) Guisnet, M.; Gnep, N. S.; Morin, S. Microporous Mesoporous Mater. 2000, 35, 47.

(e) Hoefnagel, A. J.; van Bekkum, H. Catal. Lett. 2003, 85, 7.

(9) Konakahara, T.; Kiran, Y. B.; Okuno, Y.; Ikeda, R.; Sakai, N. Tetrahedron Lett. 2010, 51, 2335.

(10) Chasset, S.; Chevreuil, F.; Ledoussal, B.; Le Strat, F.; Benarous, R. WO 2012140243 A1, 2012.

(11) Boldron, C.; Gamez, P.; Tooke, M. D.; Spek, L. A.; Reedijk, J. Angew. Chem. Int. Ed. 2005, 44, 3585.

(12) Chappell, M. D.; Conner, S. E.; Gonzalez, V. I. C.; Lamar, J. E.; Li, J.; Moyers, J. S.; Owens, R. A.; Tripp, A. E.; Zhu, G. WO 2005118542 A1, 2005.

(13) Winn, M.; Reilly, E. B.; Liu, G.; Huth, J. R.; Jae, H-S.; Freeman, J.; Pei, Z.; Zhili, X.; Lynch, J.; Kester, J.; von Geldern, T. W.; Leitza, S.; DeVries, P.; Dickinson, R.; Mussatto, D.; Okasinki, G. F. J. Med. Chem. 2001, 44, 4393.
(14) Mori, K.; Ichikawa, Y.; Kobayashi, M.; Shibata, Y.; Yamanaka, M.; Akiyama, T. J. Am. Chem. Soc. 2013, 135, 3964.

(15) Kadam, S. H.; Paknikar, S. WO 2011128018 A1, 2011.

(16) (a) Zhou, X.; Tse, M. K.; Wan, T. S. M.; Chan, K. S. J. Org. Chem. 1996, 61, 3590. (b) Enguehard, C.; Renou, J.-L.; Collot, V.; Hervet, M.; Rault, S.; Gueiffier, A. J. Org. Chem. 2000, 65, 6572. (c) Gray, M.; Andrews, I. P.; Hook, D. F.; Kitteringham, J.; Voyle, M. Tetrahedron Lett. 2000, 41, 6237. (d) Molander, G. A.; Yun, C.-S. Tetrahedron 2002, 58,1465 .

(17) Matsubara, Y.; Takekuma, S.-I.; Yokoi, K.; Yamamoto, H.; Nozoe, T. Chem. Lett. 1984, 13, 631.

(18) Sudali, A.; Rao, G. S. K. Indian J. Chem., Sect. B: Org. Chem. Incl. Med. Chem. 1989, 28, 110.

(19) Lever, J. G.; Scrivens, W. A. EP 1036782 A1, 2000.

(20) Gokhale, A.; Schiess, P. Helv. Chim. Acta 1998, 81, 251.

(21) General Procedure for Formylation Aluminium trichloride ( $4.82 \mathrm{~g}, 36 \mathrm{mmol})$ was added to a solution of phenol (33 mmol) in anhydrous $\mathrm{CH}_{2} \mathrm{Cl}_{2}(50 \mathrm{~mL})$ under argon, and the solution was stirred for $10 \mathrm{~min}$. Dichloromethyl methyl ether $(3.3 \mathrm{~mL}, 36 \mathrm{mmol})$ was added dropwise via a syringe pump $(7.7 \mathrm{~mL} / \mathrm{h})$. The reaction was left to stir for a further $10 \mathrm{~min}$ before cold $\mathrm{H}_{2} \mathrm{O}(200 \mathrm{~mL})$ was added slowly. After stirring for a further $10 \mathrm{~min}$, the organic layer was separated and washed with brine $(100 \mathrm{~mL})$ and $\mathrm{H}_{2} \mathrm{O}(150 \mathrm{~mL})$, dried over $\mathrm{MgSO}_{4}$, filtered, and concentrated to give the aldehyde which was purified by column chromatography.

2-Hydroxy-3,5-dimethylbenzaldehyde (3) Brown viscous oil; $R_{f}=0.97$ (EtOAc-PE, 1:1). IR: $v_{\max }=$ 3201, 2921, 1646, 1467, $1260 \mathrm{~cm}^{-1}$. ${ }^{1} \mathrm{H}$ NMR $(600 \mathrm{MHz}$, $\left.\mathrm{CDCl}_{3}\right): \delta=11.09(\mathrm{~s}, 1 \mathrm{H}, \mathrm{OH}), 9.81(\mathrm{~s}, 1 \mathrm{H}, \mathrm{CHO}), 7.20(\mathrm{br}$ s, $1 \mathrm{H}, \mathrm{ArH}), 7.15$ (s, $1 \mathrm{H}, \mathrm{ArH}), 2.29$ (s, $3 \mathrm{H}, \mathrm{Me}), 2.23$ (s, $3 \mathrm{H}, \mathrm{Me}) .{ }^{13} \mathrm{C}$ NMR $\left(150 \mathrm{MHz}, \mathrm{CDCl}_{3}\right): \delta=196.8(\mathrm{CH})$, $158.0\left(\mathrm{C}_{\mathrm{q}}\right), 139.1(\mathrm{CH}), 131.0(\mathrm{CH}), 128.6\left(\mathrm{C}_{\mathrm{q}}\right), 126.6\left(\mathrm{C}_{\mathrm{q}}\right)$, $119.9\left(\mathrm{C}_{\mathrm{q}}\right), 20.3\left(\mathrm{CH}_{3}\right), 15.1\left(\mathrm{CH}_{3}\right)$. HRMS (EI): $\mathrm{m} / z$ calcd for $\mathrm{C}_{9} \mathrm{H}_{10} \mathrm{O}_{2}[\mathrm{M}]^{+}: 150.0680$; found: 150.0681 .

(22) General Procedure for Trifluoromethanesulfonate Synthesis

Triethylamine ( $4.04 \mathrm{~g}, 40 \mathrm{mmol})$ was added to a solution of phenol $(13 \mathrm{mmol})$ in anhydrous $\mathrm{CH}_{2} \mathrm{Cl}_{2}(13 \mathrm{~mL})$ at $-78^{\circ} \mathrm{C}$ under argon, and the solution stirred for $30 \mathrm{~min}$.

Trifluoromethanesulfonic anhydride $(2.5 \mathrm{~mL}, 15 \mathrm{mmol}, 1.1$ equiv) was added dropwise via a syringe pump $(7.7 \mathrm{~mL} / \mathrm{h})$. The reaction was left to stir for a further $2 \mathrm{~h}$. The reaction mixture was diluted with $\mathrm{CH}_{2} \mathrm{Cl}_{2}(15 \mathrm{~mL})$ and washed with sat. $\mathrm{NaHCO}_{3}(20 \mathrm{~mL})$, brine $(20 \mathrm{~mL})$, and $\mathrm{H}_{2} \mathrm{O}(20 \mathrm{~mL})$, dried $\left(\mathrm{MgSO}_{4}\right)$, filtered, and concentrated to give the trifluoromethanesulfonate which was purified by column chromatography.

2-Formyl-4,6-dimethylphenyl trifluoromethanesulfonate (4)

Yellow viscous oil; $R_{f}=0.91$ (EtOAc-PE, 1:1). IR: $v_{\max }=$ 2883, 1701, 1598, 1407, 1207, $1136 \mathrm{~cm}^{-1} .{ }^{1} \mathrm{H}$ NMR $(600$ $\left.\mathrm{MHz}, \mathrm{CDCl}_{3}\right): \delta=10.19(\mathrm{~s}, 1 \mathrm{H}, \mathrm{CHO}), 7.62(\mathrm{~d}, 1 \mathrm{H}, J=2.0$ $\mathrm{Hz}, \mathrm{ArH}), 7.38$ (d, $1 \mathrm{H}, J=2.0 \mathrm{~Hz} \mathrm{ArH}), 2.42$ (s, $3 \mathrm{H}, \mathrm{Me})$, $2.40(\mathrm{~s}, 3 \mathrm{H}, \mathrm{Me}) .{ }^{13} \mathrm{C}$ NMR $\left(150 \mathrm{MHz}, \mathrm{CDCl}_{3}: \delta=187.4\right.$ $(\mathrm{CH}), 145.9\left(\mathrm{C}_{\mathrm{q}}\right), 139.1\left(\mathrm{C}_{\mathrm{q}}\right), 138.8(\mathrm{CH}), 132.5\left(\mathrm{C}_{\mathrm{q}}\right), 129.2$ $\left(\mathrm{C}_{\mathrm{q}}\right), 128.5(\mathrm{CH}), 118.4\left(\mathrm{q}, J=321 \mathrm{~Hz}, \mathrm{C}_{\mathrm{q}}\right), 20.9\left(\mathrm{CH}_{3}\right), 16.5$ $\left(\mathrm{CH}_{3}\right)$. HRMS (EI): $\mathrm{m} / z$ calcd for $\mathrm{C}_{10} \mathrm{H}_{9} \mathrm{~F}_{3} \mathrm{O}_{4} \mathrm{~S}[\mathrm{M}]^{+}$: 282.0174; found: 282.0174 .

(23) General Procedure for Suzuki Cross-Coupling $\mathrm{K}_{2} \mathrm{CO}_{3}(397 \mathrm{mg}, 3 \mathrm{mmol})$ and $\mathrm{PdCl}_{2}(\mathrm{dppf}) \cdot \mathrm{CH}_{2} \mathrm{Cl}_{2}(116 \mathrm{mg}$, $10 \mathrm{~mol} \%$ ) were added to a solution of aryl trifluoromethanesulfonate $(1 \mathrm{mmol})$ in THF $(25 \mathrm{~mL})$ which was left to stir for $5 \mathrm{~min}$. $\mathrm{H}_{2} \mathrm{O}$ (HPLC grade, $1.25 \mathrm{~mL}$ ) was added, followed by methylboronic acid ( $255 \mathrm{mg}, 4 \mathrm{mmol})$. The reaction was heated at reflux overnight. EtOAc $(7 \mathrm{~mL})$ was added, and the 
organic layer was separated and washed with $\mathrm{H}_{2} \mathrm{O}(2 \times 10$ $\mathrm{mL}$ ), dried over $\mathrm{MgSO}_{4}$, filtered, and concentrated to give the aldehyde which was purified by column chromatography.

2,3,5-Trimethylbenzaldehyde (1a)

Brown oil; $R_{f}=0.82$ (EtOAc-PE, 1:1). IR: $v_{\max }=2923$,

$1692,1478 \mathrm{~cm}^{-1} .{ }^{1} \mathrm{H}$ NMR $\left(600 \mathrm{MHz}, \mathrm{CDCl}_{3}\right): \delta=10.28(\mathrm{~s}$,
$1 \mathrm{H}, \mathrm{CHO}$ ), 7.46 (s, $1 \mathrm{H}, \mathrm{ArH}), 7.20$ (s, $1 \mathrm{H}, \mathrm{ArH}), 2.52$ (s, 3 $\mathrm{H}, \mathrm{Me}), 2.33$ (s, $3 \mathrm{H}, \mathrm{Me}$ ), 2.30 (s, $3 \mathrm{H}, \mathrm{Me}) .{ }^{13} \mathrm{C}$ NMR (150 $\left.\mathrm{MHz}, \mathrm{CDCl}_{3}\right): \delta=193.5(\mathrm{CH}), 138.3\left(\mathrm{C}_{\mathrm{q}}\right), 136.4(\mathrm{CH})$, $136.3\left(\mathrm{C}_{\mathrm{q}}\right), 135.4\left(\mathrm{C}_{\mathrm{q}}\right), 134.3\left(\mathrm{C}_{\mathrm{q}}\right), 130.1(\mathrm{CH}), 21.2\left(\mathrm{CH}_{3}\right)$, $20.2\left(\mathrm{CH}_{3}\right), 14.3\left(\mathrm{CH}_{3}\right)$. HRMS (EI): $\mathrm{m} / z$ calcd for $\mathrm{C}_{10} \mathrm{H}_{12} \mathrm{O}$ $[\mathrm{M}]^{+}:$148.0888; found: 148.0876 . 